\title{
CONF-980635--
}

\section{Analysis of Ripple Formation in Single Crystal Spot Welds ${ }^{\dagger}$}

\author{
M. Rappaz ${ }^{(a)}$, D. Corrigan ${ }^{(b)}$ and L. A. Boatner ${ }^{(b)}$
}

(a) Laboratoire de métallurgie physique Ecole Polytechnique Fédérale de Lausanne MX-G, CH-1015 Lausanne, Switzerland

(b) Oak Ridge National Laboratory

Solid State Division, P. O. Box 20

Oak Ridge, TN 37830, USA

\begin{abstract}
Stationary spot welds have been made at the (001) surface of $\mathrm{Fe}-15 \% \mathrm{Ni}-15 \% \mathrm{Cr}$ single crystals using a Gas Tungsten Arc (GTA). On the top surface of the spot welds, very regular and concentric "ripples" were observed after solidification by differential interference color microscopy. Their height (typically $1-5 \mu \mathrm{m}$ ) and spacing (typically $\sim 60 \mu \mathrm{m}$ ) decreased with the radius of the pool. These ripples were successfully accounted for in terms of capillary-wave theory using the fundamental mode frequency $f_{0}$ given by the first zero of the zero-order Bessel function. The spacing $d$ between the ripples was then equated to $v_{s} / f_{0}$, where $v_{s}$ is the solidification rate. From the measured ripple spacing, the velocity of the pool was deduced as a function of the radius, and this velocity was in good agreement with the results of a heat-flow simulation.
\end{abstract}

\section{DTC QUALTTY INSPECTHES}

$\dagger$ This research has been supported by the National Areonautics and Space Administration Microgravity Sciences and Applications Division, Project \# 962-25-08-23 under NASA Contract \#H-13059 with Oak Ridge National Laboratory. Oak Ridge National Laboratory is managed for the U.S.D.O.E under contract DE-AC05960R22464 with Lockheed Martin Energy Research Corp. 


\section{Introduction}

The formation of ripples during welding or laser remelting is a problem that has been known for many years but which is yet not fully understood. Several mechanisms have been invoked to explain the ripple formation, most of them being related in some way to capillarity. Ripples are sometimes associated with what has been called "low-velocity bands" $[1,2]:$ in this case, the whole melt pool does not seem to move with a steady-state speed and bands can be seen in transverse or longitudinal sections of the weids. In some other cases, ripples appear only at the top surface without in-depth correlation.

Kotecki et al [3] studied the formation of ripples in GTA (Gas Tungsten Arc) spot welds. They observed concentric ripples after solidification which they attributed to oscillations of the liquid surface. However, their analytical model of natural oscillation in terms of a vibrating drum membrane is over-simplistic. More recently, Postacioglu et al [4] made a detailed calculation of natural and forced oscillations of a melt pool with particular attention to keyhole formation.

In a now classical paper, Anthony and Cline [5] took a totally different approach to explain ripple formation during continuous laser welding. They considered the simple situation of a one-dimensional Marangoni flow induced by the surface tension gradient and calculated the pressure difference along the surface. They converted then this pressure gradient into a metallostatic head difference but ignoring the capillarity contribution (i.e., the Laplace force). Nakane [6] has made a detailed experimental investigation of ripples formed during GTA welds, under various conditions. He made a clear distinction between macroripples, which occur at low speed and propagate in depth of the weld, and microripples appearing at higher speed.

In the present work [7], spot welds were made in austenitic single crystals in order to study the formation of microstructure and ripples. It is shown that the smail ripples that form under these conditions can be analyzed using the formalism of standing capillary waves [8]. This formalism is summarized in section 3 after the experimental section. In section 4, the modeling of fusion and resolidification of the molten pool is briefly presented. Finally, the results are presented and discussed in section 5 .

\section{Experiment}

The spot welds were made on $\mathrm{Fe}-15 \mathrm{wt} \% \mathrm{Ni}-15 \% \mathrm{Cr}$ single crystal specimens. The procedure used to grow and to prepare these specimens is identical to that used to perform autogeneous welds [1] and thus will be only briefly summarized here. After being grown using a Czochralski technique, the single crystals were oriented by X-ray diffraction and then cut in order to obtain thin plates (typically $4 \mathrm{~mm}$ thick and $30 \mathrm{~mm}$ in diameter) bounded by (100) flat surfaces. These surfaces were then polished and ultrasonically cleaned in an acetone bath.

The spot welds were made at the center of the discs using a Gas Tungsten Arc (GTA) process, under the protection of a He-Ar shielding gas. The standard conditions were typically $80 \mathrm{~A}$ and $10 \mathrm{~V}$ applied for $10 \mathrm{~s}$ by a continuous power source. The resulting spot weld at the end of the heating stage was typically hemispherical in shape, with a diameter of about $6 \mathrm{~mm}$. The power was then stopped and the molten pool solidified typically in about $0.5 \mathrm{~s}$.

After solidification, the top surface of the spot welds was directly observed without any preparation by optical microscopy, sometimes using a differential interference color adapter. This last equipment could reveal very nicely the small ripples formed at the surface. The surface profiles were measured along radial lines using a profilometer with a diamond tip. 


\section{DISCLAIMER}

This report was prepared as an account of work sponsored by an agency of the United States Government. Neither the United States Government nor any agency thereof, nor any of their employees, makes any warranty, express or implied, or assumes any legal liability or responsibility for the accuracy, completeness, or usefulness of any information, apparatus, product, or process disclosed, or represents that its use would not infringe privately owned rights. Reference herein to any specific commercial product, process, or service by trade name, trademark, manufacturer, or otherwise does not necessarily constitute or imply its endorsement, recommendation, or favoring by the United States Government or any agency thereof. The views and opinions of authors expressed herein do not necessarily state or reflect those of the United States Government or.any agency thereof. 


\section{Capillary wave theory and ripple formation}

This section of the paper addresses the mathematical formulation of standing capillary waves and the resulting ripples that form during solidification. The model is derived from the formalism of Landau and Lifshitz [8] : it is based upon the Navier-Stokes equation with appropriate boundary conditions and is much simpler than the formalism derived by Postacioglu et al [4] using an energy principle.

The basic assumptions of the model are as follows : $i)$ the fluid is incompressible, non-viscous and irrotational (i.e., rot $\mathbf{v}=0$ ) ; ii) the hemispherical melt pool of radius $R$ and height $h$ is replaced by a cylindrical shape with a flat bottom having the same dimensions (see Figure 1) ; iii) the wave is totally constrained at the bottom and lateral surface of the molten pool, including the triple point between the liquid, solid and air (i.e., zero velocity of the fluid at $r=R$ and $z=-h$, see Figure 1 ) ; iv) only the vertical component of the velocity, $v_{z}$, is considered. If these hypotheses are reasonable in the axisymmetric situation and the small amplitude wave considered here, the question of the constraint introduced at the triple point will be discussed after presenting the theory. Neglecting the viscous term, the Navier Stokes equation for the vertical component of the velocity, $v_{z}$, can be written as :

$$
\rho \frac{d v_{z}}{d t}=\rho \frac{\partial v_{z}}{\partial t}+\rho v_{z} \frac{\partial v_{z}}{\partial z}=-\frac{\partial P}{\partial z}-\rho g
$$
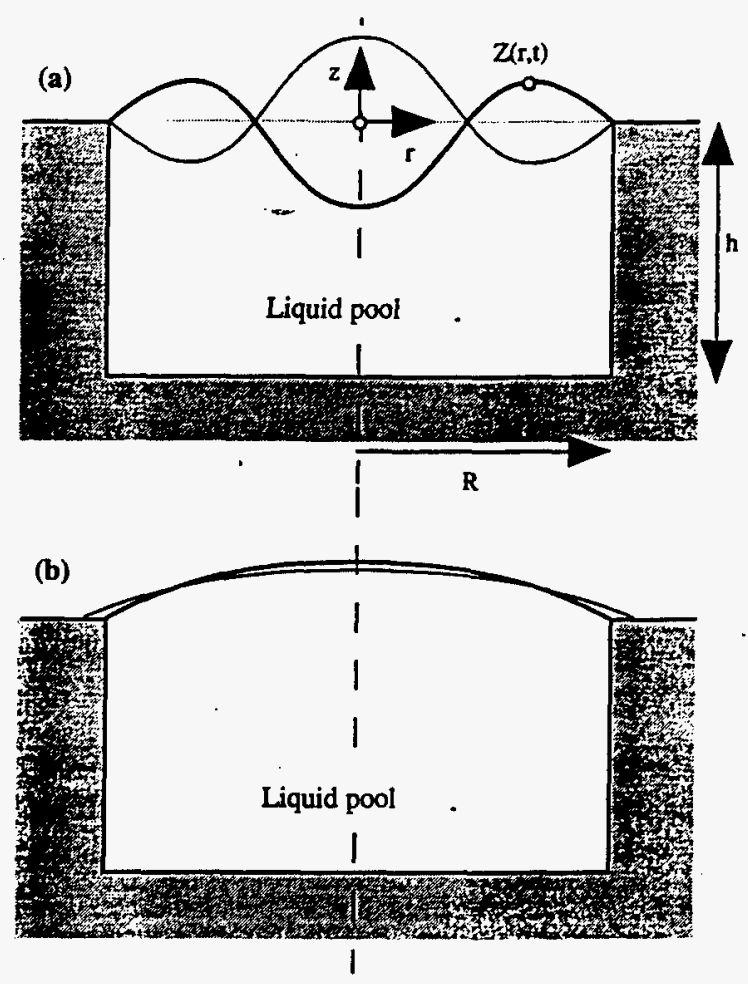

Figure 1: Schematic of the idealized situation used in the capillary wave model when the triple point is constrained not to move (a). The first mode which conserves mass under this condition corresponds to the second zero of the first-order Bessel function, $J_{0}$. If the triple point is allowed to move laterally $(b)$, the first mode then corresponds to the first zero of $J_{o}$

In this equation, $\rho$ is the fluid density, $\mathrm{P}$ is the pressure and $-g$ is the vertical component of gravity (assumed to be opposite to the zdirection). Since the fluid is irrotational (i.e., rot $\mathbf{v}=0$ ), this means that there is a velocity potential, $\phi$, such that $\mathbf{v}=\operatorname{grad} \phi$. Inserting this relation in eq. (1) and integrating with respect to $\mathrm{z}$, one gets :

$P-P_{a}=-\rho g z-\rho \frac{d \phi}{d t}$

On the other hand, the difference between the dynamic pressure in the liquid, $P$, and the atmospheric pressure, $\mathrm{P}_{\mathrm{a}}$, must be compensated by the surface tension of the liquid surface. Under the assumption of small displacements, this means that :

$P-P_{a}=-\gamma\left[\frac{\partial^{2} Z}{\partial r^{2}}+\frac{1}{r} \frac{\partial Z}{\partial r}\right]$

where $\gamma$ is the surface tension of the liquid and $Z(r, t)$ the position of its top surface as a function of the radial distance and time. Please note that the curvature has been approximated by the Laplacian of the liquid position, $-\Delta Z$. This equation can be derived with respect to time so as to introduce the vertical velocity of the top surface, and thus 
the velocity potential. Combining this equation with equation (2) also derived with respect to time, one finally gets an equation governing the potential $\phi(r, z, t)$ :

$$
\rho g \frac{\partial \phi}{\partial z}+\rho \frac{\partial^{2} \phi}{\partial t^{2}}-\gamma \frac{\partial}{\partial z}\left[\frac{\partial^{2} \phi}{\partial r^{2}}+\frac{1}{r} \frac{\partial \phi}{\partial r}\right] \quad \text { at } z=Z
$$

Note that this equation is only valid at the top surface of the liquid and in reality is a boundary condition for the velocity potential $\phi$. The other equations governing the velocity potential are :

$$
\text { In the volume: } \Delta \phi=\frac{1}{r} \frac{\partial}{\partial r}\left[r \frac{\partial \phi}{\partial r}\right]+\frac{\partial^{2} \phi}{\partial z^{2}}=0
$$

On the constrained surfaces of the cylinder, the velocity must be zero when a no-slip condition is imposed. Because only the vertical component of the velocity field, $v_{z}$, has been considered, the following boundary conditions are imposed at the solid-liquid interface :

$$
\mathrm{v}_{\mathrm{z}}=\frac{\partial \phi}{\partial \mathrm{z}}=0 \quad \text { at } \mathrm{r}=\mathrm{R} \text { and } \mathrm{z}=-\mathrm{h}
$$

A solution of Eqs (4-6) is provided in Ref. [7]. for the case of a cylindrical molten pool. In the case where the gravity term can be neglected in Eq. (4), the eigenmodes of the cylindrical molten pool are given by :

$$
\mathrm{f}_{\mathrm{n}}=\frac{1}{2 \pi} \sqrt{\frac{\gamma}{\rho}\left(\frac{\left.\lambda_{\mathrm{an}}\right)^{3}}{\mathrm{R}}\right)^{\tanh \left(\frac{\lambda_{\mathrm{on}}}{\mathrm{R}} h\right)}}
$$

where $\lambda_{0, n}$ is the $n-t h$ zero of the zero-order Bessel function, $J_{0}$, and $f_{n}$ the associated oscillation frequency of the molten pool. The first mode of vibration $\left(\lambda_{0,1}=2.405\right)$, called here the "breathing" mode, would correspond to a uniform displacement of the pool surface (i.e., all the points of the free surface go up and down at the same time). Therefore, this mode does not satisfy mass conservation if the triple point is totally constrained (i.e., if the point at the junction of the solid, liquid and air is not allowed to move slightly). However, when the wetting angle at the free surface of the pool is small, which is the case for most metals as the liquid wets the solid very well, lateral displacement of this triple point can be envisaged (see Figure $2 b$ ). Although the present model does not strictly apply to such a situation, it will be assumed that Eq. (7) still gives a reasonable estimate of the fundamental oscillation frequency of the molten pool. This point is further discussed in Ref. [7].

The boundary condition at the triple point being relaxed, this point can move back and forth (and up and down). In this case, the situation is similar to the basic oscillation mode of a wave that would break down near the "shore" of the pool, while at the same time the pool solidifies from the edge. This will finally leave ripples on the frozen surface separated by a spacing, $d$, given by :

$$
d(R)=\frac{v_{s}(R)}{f_{0}(R)}=v_{s}(R) \tau_{0}(R)
$$

where $v_{s}$ is the solidification rate. The basic mode of vibration, $f_{0}$, given by the first zero of $J_{0}$, has been assumed in the present equation, $\tau_{0}$ being the time period of the mode. This situation is illustrated in Figure 2. If the liquid surface had a steady shape, the triple point would move almost linearly. However, such is not the case due to the capillary oscillations. At time $t$, the liquid surface is assumed to have the maximum lateral extent, and thus the minimal angle, $\theta_{\min }$, with the surface of the solid. One period, $\tau_{0}$, of the oscillation later, the triple point has moved a 
Table 1 Thermophysical properties of the $\mathrm{Fe}-15 \% \mathrm{Ni}-15 \% \mathrm{Cr}$

$\begin{array}{llrl}\text { Volumetric latent heat of fusion } & & 1.910^{9} & \mathrm{Jm}^{-3} \\ \text { Volumetric specific heat at } & \mathrm{T}=200^{\circ} \mathrm{C} & 3.1610^{6} & \mathrm{Jm}^{-3}{ }^{\circ} \mathrm{C}^{-1} \\ & \mathrm{~T}=1400^{\circ} \mathrm{C} & 5.4510^{6} & \\ & \mathrm{~T}=200^{\circ} \mathrm{C} & 14 \mathrm{Wm}^{-1}{ }^{\circ} \mathrm{C}^{-1} \\ \text { Thermal conductivity at } & \mathrm{T}=1405^{\circ} \mathrm{C} & 28 & \\ & \mathrm{~T}>1425^{\circ} \mathrm{C} \text { (liquid) } & 280 & \\ & & 1425 & { }^{\circ} \mathrm{C} \\ \text { Liquidus temperature } & 20{ }^{\circ} \mathrm{C}\end{array}$

\section{Results and discussion}

Figure 3 shows the interference color picture of a weld made under standard conditions on the (001) surface of a single crystal stainless steel specimen. The diameter of the molten pool at the end of heating $(10 \mathrm{~s})$ was about $6 \mathrm{~mm}$. This value is in close agreement with the result of the simulation which predicts a molten pool of $6.6 \mathrm{~mm}$ in diameter after heating.

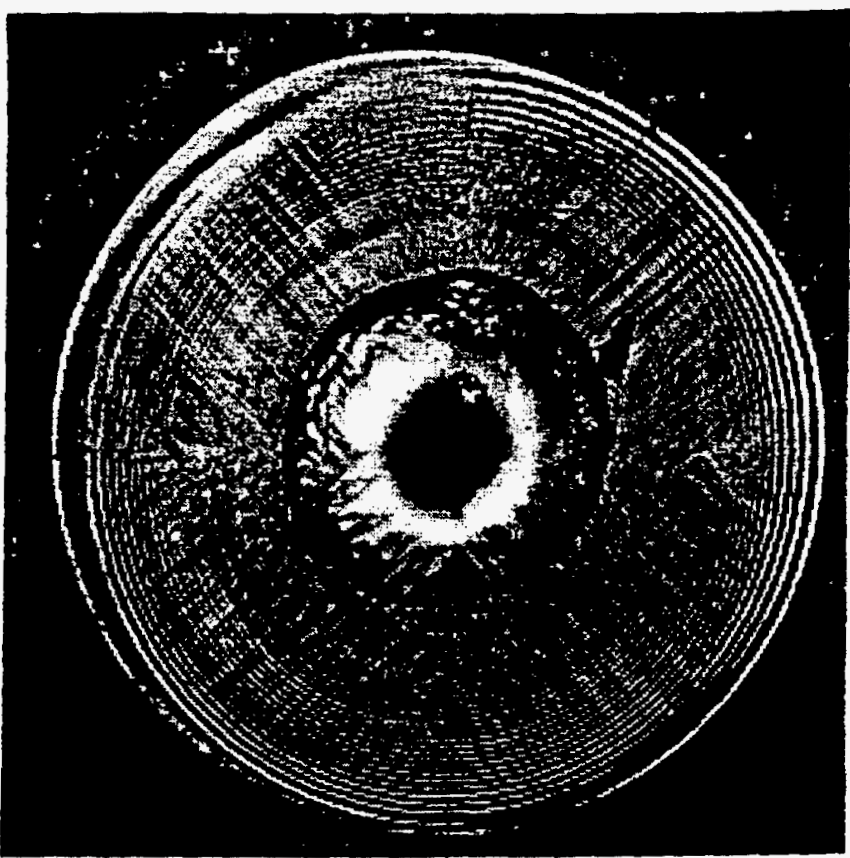

Figure 3 : Differential interference color picture of the top surface of a spot weld made under standard conditions.
During solidification, nearly perfectly circular and concentric ripples form and appear as successive white and black rings in this macrograph. They correspond to slightly raised and depressed parts of the solidified surface, respectively. As can be seen, their spacing decreases as the radius of the pool decreases. Equation (8) predicts that the spacing of the ripples should vary as the product of the solidification rate times the period of the capillary wave. As will be shown in more detail later in this section, the solidification rate is fairly constant up to middistance of the pool and thus this dependence of the ripple spacing is mainly due to the $\mathrm{R}^{3 / 2}$ dependence appearing in Eq. (7) (providing the depth over width

ratio of the pool, $\mathrm{h} / \mathrm{R}$, is fairly constant). Near the center of the weld, the ripples are so narrowly spaced that they can hardly be seen. Please note that the ripples are more regular in the bottom part of the pool, whereas they look slightly blurred in the upper one. Precisely in this last region, the edge of the weld does not seem very well defined : this is due probably to the reminiscence of the heating stage which clearly induced some fluctuations in the weld pool position (instability of the arc-pool system).

From the contrast seen in Figure 3, it also appears that the overall shape of the solidified surface is significantly higher near the periphery of the spot weld, then goes down close to the center (dark wide ring located near $R=1 \mathrm{~mm}$ ), then goes up again (light ring). A cavity seems to have formed at the center (dark circle). This overall variation of the solidified surface has been anaiyzed in Ref. [7] in terms of solidification shrinkage. Superimposed to the contrast 
distance $d=v_{s} \tau_{0}$. At the half period, $\tau_{0} / 2$, the pool has the minimal lateral extent (or maximal pool height at the center) and the angle between the liquid and solid surfaces, $\theta_{\max }$, has now a maximum value. As a consequence, the triple point is slightly depressed with respect to the position it would have (dashed line) if the liquid surface shape was stationary. The trajectory of the triple point has a sinusoidal shape instead of a straight line as indicated in this figure. In summary, the solidified surface near the rim of the pool has a maximum height when the liquid pool has a minimum level at the center, and vice versa.

Since both $v_{s}$ and $f_{o}$ are a function of the radius of the pool, $R$, the spacing between the ripples also depends on $\mathrm{R}$. As can be seen in Eq. (8), it is necessary to deduce a relationship between the growth rate and the radius of the pool. The next section outlines a heat-flow calculation based upon a purely-diffusive model with latent heat release which allows to obtain this velocity.

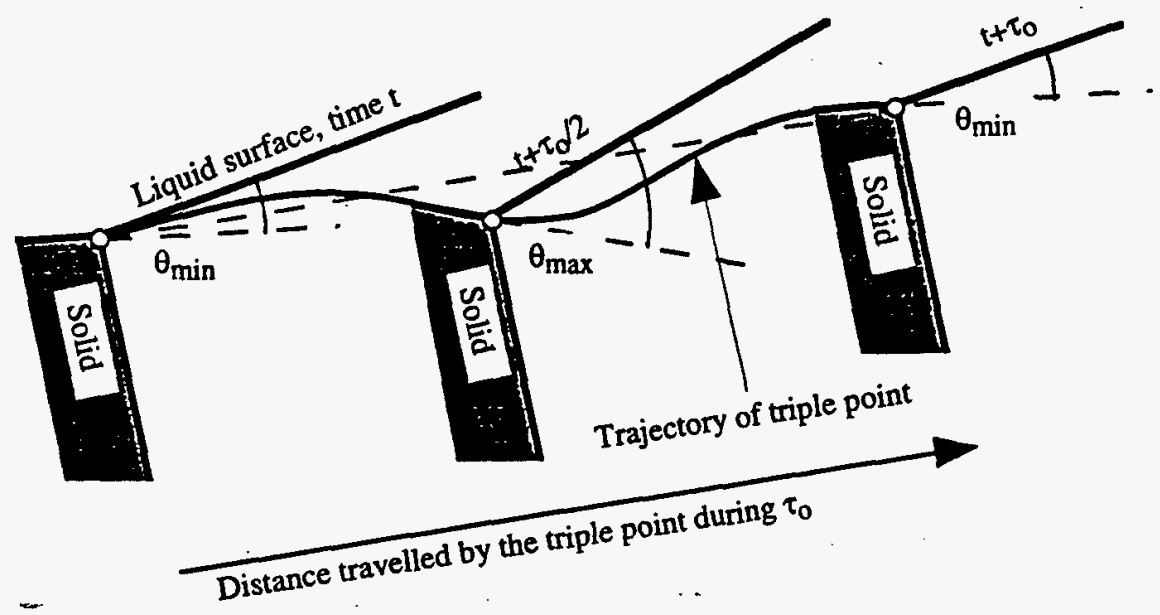

Figure $2:$ Schematics of the mechanism involved in the formation of ripples, with the vibration mode of Figure $1 b$.

\section{Hemispherical pool fusion and solidification}

The formation of the molten pool and its subsequent resolidification was modeled using the finite element solidification code 3-mos, developed at the Ecole Polytechnique Fédérale de Lausanne [9]. Although this package can include convection, solute transport or microstructure calculations, only the heat-flow part was used. The heat flow equation with latent heat release was solved for an axisymmetric situation using an enthalpy method.

Since the small specimen was attached onto a base plate of copper to ensure good electrical and thermal contact, the simulation was instead performed for a semi-infinite domain (in the present case a cylinder $20 \mathrm{~cm}$ in diameter and $4 \mathrm{~cm}$ thick). This avoided the problem of specifying an arbitrary heat transfer coefficient between the plate and the copper chill. The power $(800 \mathrm{~W})$ was distributed evenly over an arbitrary surface of $4 \mathrm{~mm}$ of diameter and applied for a period of $10 \mathrm{~s}$ (the experimental heating period). The thermophysical properties of the specimen used in the heat flow computations are indicated in Table 1.

The computation was performed by setting adiabatic conditions at the external boundaries, except of course under the arc. As the diffusion distance over a period of $10 \mathrm{~s}$ is only about $7 \mathrm{~mm}$, setting a non-zero heat transfer coefficient on these surfaces has no effect. Similarly, radiation and convection on the welded surface are negligible with respect to the heat sink provided by the copper chill (or the bulk of the specimen in the present case). The initial temperature was set to room temperature. The velocity at the top surface was then calculated from the evolution of the molten pool shape. 
associated with the uneven surface of the weld are faint straight lines at $\pm 45 \mathrm{deg}$ with respect to the horizontal. They correspond to the $[ \pm 100]$ and $[0 \pm 10]$ directions of the dendrite trunks growing nearly parallel to the surface. As was shown for autogeneous welds made on single crystals [1], the dendrite trunks which are selected by the growth competition process are those which have the minimal velocity or minimal undercooling. In the case of the non-stationary spot welds investigated here, the thermal gradient and velocity of the liquidus isotherm are approximately radial. Therefore, within each quadrant of the solid-liquid interface defined by the (110) and (i10) planes, a specific $<100>$ dendrite trunk variant grows near the top surface.

The profilometry measurement of the weld shown in Figure 3 is presented in Figure 4. It has been recorded for a region near the periphery of the weld, along a radial direction, and the scales are indicated in the figure caption. As already observed on the macrograph of Figure 3, the spacing between the ripples becomes smaller as the radius of the pool decreases. The amplitude of the ripples is typically $1 \mu \mathrm{m}$ and also decreases with the radius. The overall shape of the surface is remarkably flat, less than $5 \mu \mathrm{m}$ variation over the $1.6 \mathrm{~mm}$ analyzed distance.

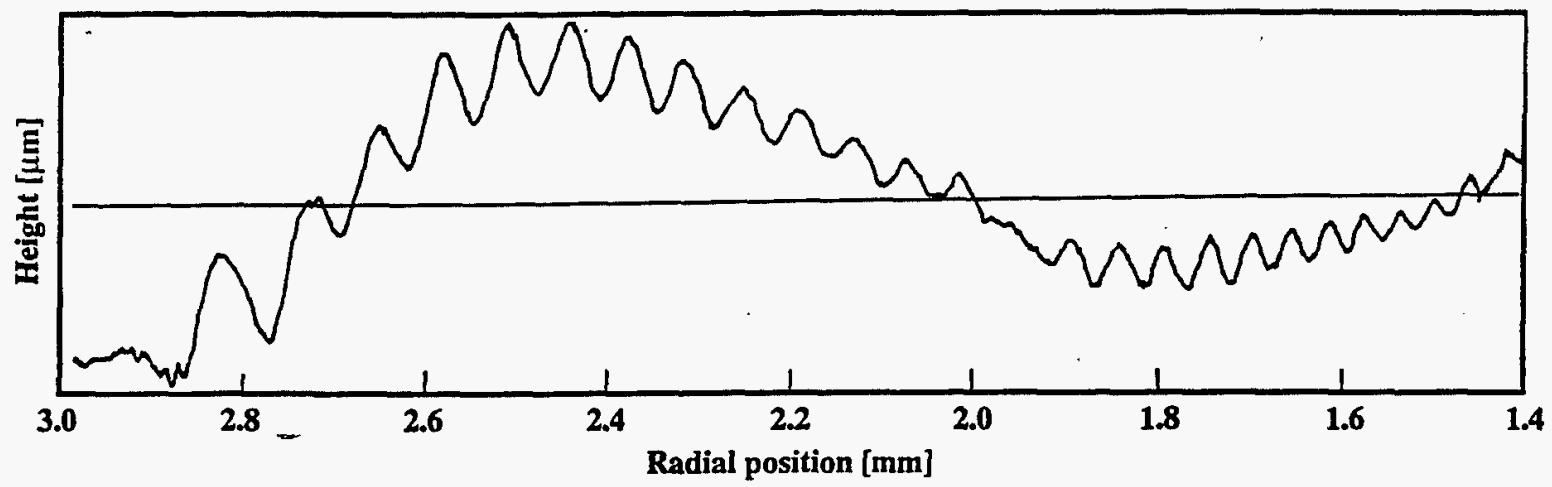

Figure 4 : Profilometry measurement along a radius of the weld shown in Figure 3. The horizontal scale is $1.6 \mathrm{~mm}$ starting from the edge of the weld, the vertical displacement measured on the surface is $5.6 \mu \mathrm{m}$.

Using the ripple spacing shown in Figure 4 and the model of capillary wave presented in section 3 (Eq. (7)), the actual velocity of the pool can be computed as a function of its radius (Eq. (8)). The breathing mode $\left(\lambda_{0,1}=2.405\right)$ was used for a "hemispherical" pool $(h / R=1)$ with a surface tension $\gamma=1.9 \mathrm{~N} / \mathrm{m}$. The results obtained in this way are shown in Figure 5 (filled squares), together with another set of measurements (filled triangles) performed on the same weld but along another radial direction, perpendicular to that used in Figure 4. As can be seen, the data are fairly consistent. A least-squares fit of the type $v(R)=A R^{-n}$ was then made independently for each set of data. The result is displayed with dashed lines in Figure 5 with an indication of the fitted parameters. The prediction of the heat flow calculation is shown with open circles in this figure. The computation which was done independently from the ripples analysis shows that the results are remarkably consistent. In both analyses, the several parameters were not adjusted to obtain this agreement, but were taken from the available literature. The continuous curve shown in Figure 5 is also a fit $v(R)=A R^{-n}$ made on the data points obtained from the heat flow calculation over the same range of radius.

Near the center of the weld, the solidification rate calculated with the heat flow model does not follow a $\mathrm{R}^{-\mathrm{n}}$ dependence, but unfortunately the ripples are no longer visible in this region. Nevertheless, the solidification times, $t_{s}$, calculated from the ripples analysis $\left(t_{s}=0.61 \mathrm{~s}\right.$ or $0.54 \mathrm{~s}$ for series $\# 1$ and $\# 2$, respectively) or from the heat flow computation $\left(t_{s}=0.59 \mathrm{~s}\right)$ are in very good agreement. In the first analysis (ripples), this time was obtained from the integration of the fit $v(R)=d R / d t=A R^{-n}$, from 0 to $3 \mathrm{~mm}$, whereas that mentioned for the heat flow analysis directly comes from the computation. 


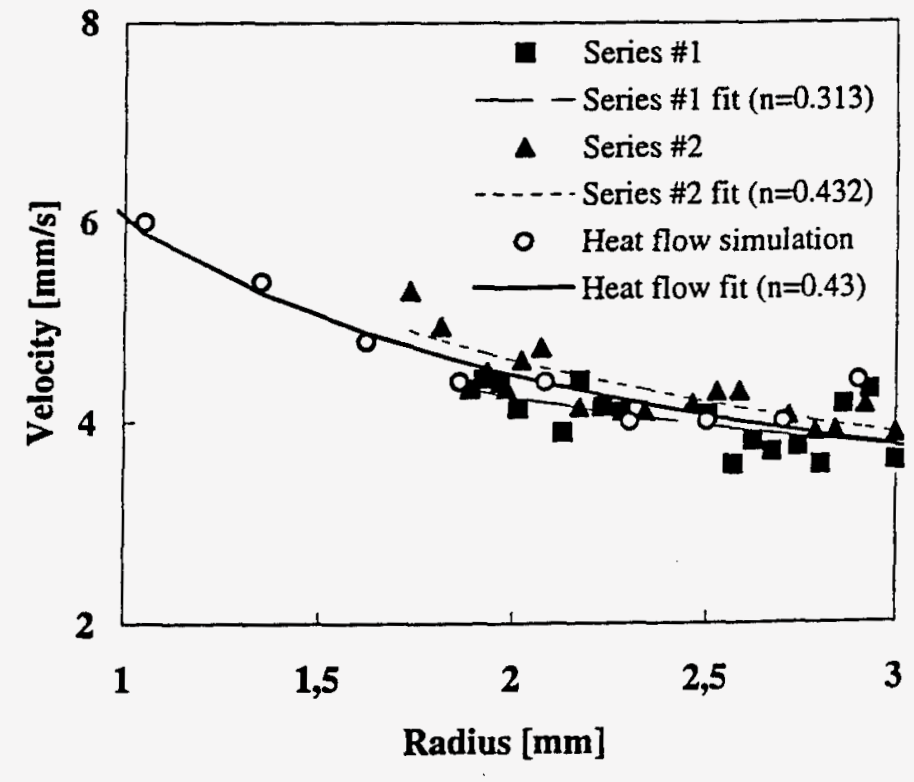

Figure 5: Solidification rate as a function of the radius of the molten pool, as deduced from the measured ripple spacing and the model described in section 3. The squares and diamonds correspond to two sets of measurements. on which dotted curves of the type $v=A R^{-n}$ have been fitted using a leastsquares method. The continuous curve shown in this figure has been calculated independently with the simple model of weld pool solidification outlined in section 4.

\section{Conclusion}

Based upon the capillary wave theory, a simple analysis of ripple formation has been performed. It has been shown that the ripple spacing is the result of the product of the solidification rate and of the period of the melt pool oscillation. The solidification rate deduced from the analysis of the ripple spacing has been found to be in good agreement with that deduced independently from heat flow computations.

\section{Acknowledgments}

The authors wish to thank Jean-Luc Desbiolles for his help in the heat-flow analysis with the software 3-mos.

\section{References}

1. M. Rappaz, S. C. David, J. Vitek and L. A. Boatner, Met. Trans. 21A (1990) 1767.

2. M. Gremaud, M. Carrard and W. Kurz, Acta Metall. Mater. 39 (1991) 1431.

3. D. J. Kotecki, D. L. Cheever and D. G. Howden, Welding Research Suppl. (August 1972) 386.

4. N. Postaciogiu, P. Kapadia and J. Dowden, J. Phys. D, Appl. Phys. 22 (1989) 1050.

5. T. R. Anthony and H. E. Cline, J. Appl. Phys. 48 (1977) 3888.

6. K. Nakane, Ripple Formation on the Surfaces of GTA Welding Beads, Memoirs School Sc. Engng., Waseda Univ. 44 (1980) 1.

7. L. A. Boatner, D. Corrigan, S. A. David, M. Rappaz and J. Sipf, Study of Ripple Formation in Single Crystal Spot Welds, in preparation.

8. L. Landau and E. Lifchitz, Mécanique des fluides (Editions MIR, Moscou, 1970).

9. Ph. Thévoz, M. Rappaz, J. L. Desbiolles, in Light Metals, Ed. Ch. M. Bickert (The Minerals, Metals and Materials Soc., 1990), p. 975. 
M98000897

Report Number (14) ORNL/CP_- 94924

CONF- 980635 -

Publ. Date (11) 199710

Sponsor Code (18) DOE/ER: NASA , XF

UC Category (19) UC-404; OO2, DOE/ER

DOE 